\title{
CONSTRUCTION OF STANDARDISED SIMULATED ROOT CANALS IN RESIN BLOCKS FOR PRE-CLINICAL TEACHING
}

\author{
W.K.M. Soo, Y.L. Thong. Construction of standardised \\ simulated root canals in resin blocks for pre-clinical \\ teaching. Annal Dent Univ Malaya 2002; 9: 7-10.
}

Original Article

W.K.M. Soo ${ }^{1}$ and Y.L. Thong ${ }^{2}$
${ }^{1}$ Graduate Student
${ }^{2}$ Associate Professor
Department of Conservative Dentistry,
Faculty of Dentistry,
University of Malaya
50603 Kuala Lumpur
Corresponding author - Assoc. Prof. Thong Yo Len

Webber (4) compared the incidence of hourglass shaped root canals in prepared canals from extracted teeth and in prepared canals formed in clear resin blocks. The non-significant difference between the two groups indicated that the RSC was a valid model for studying prepared canal shapes. However, the workers commented that unlike the fine dentinal debris formed during canal preparation in extracted teeth, the resin debris produced during preparation of simulated canals was in short strands, which tend to block the apical portion of the canal. Other limitations of RSC include lack of an apical constriction and the difference in surface texture of resin compared to dentine.

Commercial products with standard straight and curved canals are available (Dentsply Maillefer, Ballaigues, Switzerland; Trimunt Corporation, Kyoto, Japan; Frasaco, Tettnang, Germany), but tend to be more expensive than the ones made in dental institutions for pre-clinical teaching (Figure 1). Some of these RSC have stained or coloured canal walls,

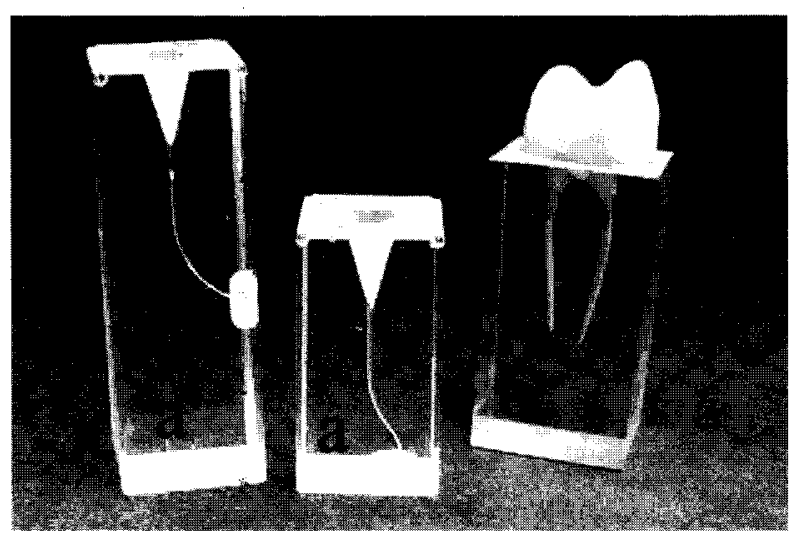

Figure 1: Commercial resin blocks

a. Manufactured by Dentsply Maillefer, Ballaigues, Switzerland

b. Manufactured by Trimunt Corporation, Kyoto, Japan. 


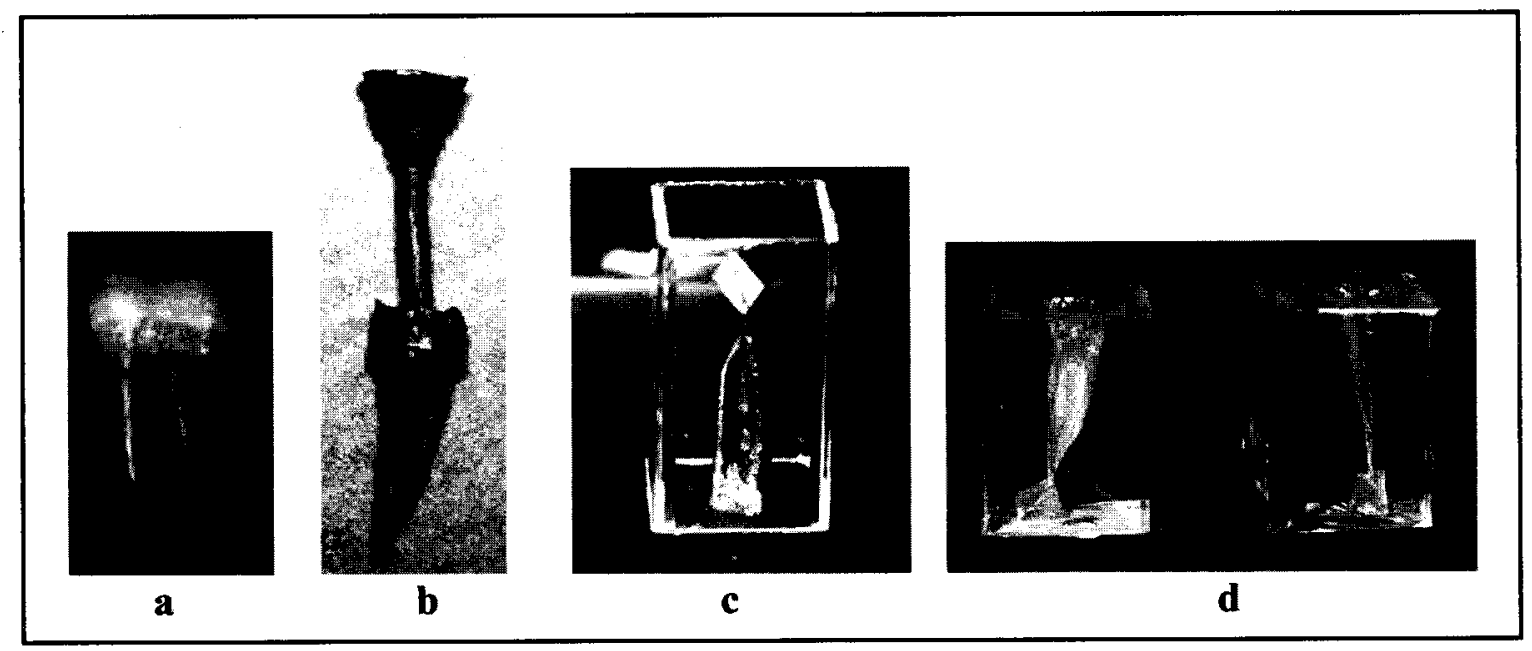

Figure 2: Construction of simulated C-shaped canals

a. A mandibular second molar with C-shaped root and single continuous C-shaped canal (Canal Type I) is used to construct resin simulated C-shaped canals.

b. A metal replica of the prepared C-shaped canal is obtained.

c. The metal canal replica is insertod into a softened modelling wax base and a prepared cuvette is placed over it.

d. Polymerised resin blocks with simulated canals after removal of metal replicas.

which allow progressive canal shaping to be visualised. There are also commercially available RSC with simulated lateral canals (Kilgore International Inc., MI, USA). However, RSC of irregular shaped canals have to be specially constructed using a replica of the canal system of an actual tooth (Figure 2).

The following describes the method of construction of RSC in the Faculty of Dentistry, University of Malaya, used for both pre-clinical teaching and research, modified from techniques described in the literature $(1,5,6,7)$. Alternative materials and procedures recommended in the literature are also described.

\section{CONSTRUCTION OF RESIN SIMULATED CANALS}

\section{Canal Template}

Simulated straight or curved canals with round cross-sections are constructed using silver points, sizes 10 to 20 , or stainless steel finger spreaders size FineFine, as templates. Prior to the embedding process, to produce standardised curved RSC, the points are curved by hand against the curved surface of an aluminium plate that has the required degree of curvature (Figure 3). Alternatively, to obtain curved RSC with standardised angles and positions of curve for use in research, Alodeh et al. (8) described a canal former in which silver points were slipped into channels prepared on the surface of an acrylic block to form smooth curves on the points. Silver points may be annealed prior to use by placing the precurved points in a preheated oven at $200^{\circ} \mathrm{C}$ for two hours, as suggested by Lim and Webber (4), in order to allow them to retain any curve which is imparted to them

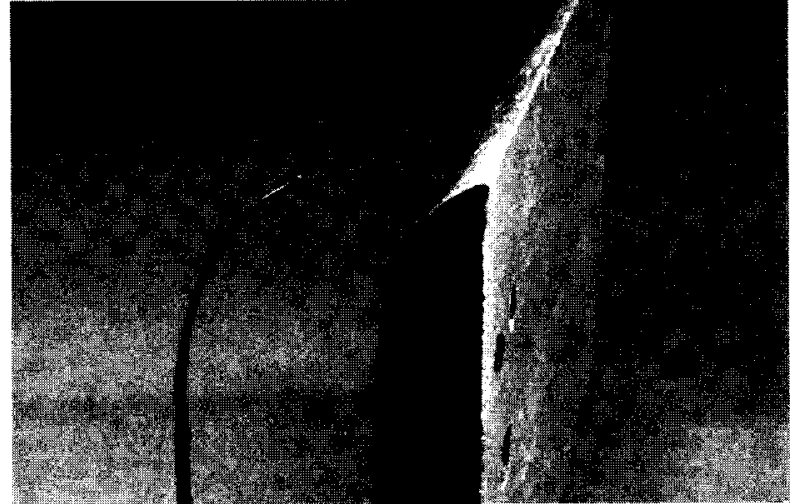

Figure 3: A silver point is curved by hand against the curved surface of an aluminium plate.

and to facilitate removal of points from polymerised resin (6). A silver point with a double curvature may be used to produce an S-shaped canal. RSC with canal configurations other than a single canal may be constructed by using two curved silver points to form the whole canal system, for example Canal Type II (21) and Canal Type V (1-2).

To construct simulated irregular shaped canals, for example C-shaped canals, a replica of the canal system has to be obtained. Injecting resin into a canal system and then dissolving the tooth in acid to obtain the resin canal replica has been described in the literature ( 7 , 9). First, an extracted tooth is selected and an access cavity prepared. In Figure $2 a$, the C-shaped canal of a mandibular second molar is cleaned and shaped prior to replicating the canal system. A polyester casting resin (Miracon (M) Sdn Bhd, Malaysia) is injected into the canal space using manual pressure on a Luer-lok syringe with a 25 gauge needle fitted to it. 
During the first few seconds of injection, the apical foramen is blocked with a gloved finger and then removed. The injection continues until excess resin emerges from the apical foramen. A vibrator may be used to facilitate the flow of resin into the canal space. After leaving the specimen for 24 hours at room temperature for solidification of resin, the tooth is decalcified in $35 \%$ aqueous nitric acid until total dissolution of tooth substance. The resin canal replica is invested in cristobalite (Kerr Corporation, Romulus, MI, USA) and burned out at $750^{\circ} \mathrm{C}$ in a furnace (Combilabor, Heraeus Hanau, Germany) and then cast using a semi-precious alloy (Palliag ${ }^{\circledR} \mathrm{M}$, Degussa AG, Hanau, Germany) into a metal replica (Figure 2b). This metal replica is used as a canal template to construct RSC. All undercuts have to be removed to facilitate the construction of RSC.

To maintain apical canal patency, a small cube of agar hydrocolloid, silicone or wax is attached to the apical tip of the canal template. In order to facilitate its removal, a thin layer of lubricating oil (WD-40, WD-40 Company (Australia) PTY. LTD., Australia) may be applied to the template prior to the embedding process.

\section{Clear Resin}

Ideally, the resin used for constructing RSC should possess the same hardness as dentine, does not shrink upon polymerisation or react with the mould and is heat-resistant. It should also be of low viscosity, easily manipulated and have a short setting time. A variety of resins have been used for this purpose in the Faculty, for example, a dimethacrylate resin (LR White, London Resin Company, England), a low viscosity epoxy resin (Stycast 1266, Emerson \& Cuming, Belgium) and an epoxy resin (Mirapox, Miracon (M) Sdn. Bhd., Malaysia). The disadvantage of LR White is that it has to be stored and transported at $4{ }^{\circ} \mathrm{C}$ to prevent premature polymerisation. Dummer et al. (6) stated that Stycast 1266 is softer than dentine. No investigation has been carried out to determine the hardness of Mirapox. The resin has to be mixed and prepared according to the manufacturer's instructions. It is essential to use the resin base and catalyst ratio recommended by the manufacturer because changes in the ratio can affect the surface hardness of resin. It is also very important to vacuum-deair the mixture to remove any air voids that are incorporated during manipulation to produce high quality RSC.

\section{Mould}

It is necessary to use a mould into which the resin is poured and allowed to set. Disposable transparent spectrophotometer cuvettes of $3.5 \mathrm{ml}$ (Delta Analytical Instruments, PA, USA) with the closed bottom portions removed are used as moulds. The cuvettes form the external walls of the polymerised resin block. Alternatively, a silicone mould, developed by taking an impression of a previously manufactured resin model may be used, as suggested by LaTurno et al. (3).

\section{Embedding process}

The coronal portion of the canal template or replica is inserted into a softened modelling wax base that is allowed to set in a shallow tray, so that the template protrudes above the wax surface. A cuvette is placed over the canal template with the two opposing sides of the cuvette parallel to the mesial and distal surfaces of the template. Figure $2 \mathrm{c}$ shows a metal Cshaped canal replica with a cuvette placed over it. Wax is used to seal the base of the cuvette to prevent leakage of resin. The resin is vacuum mixed and then poured into the moulds. For the irregular canal template, resin may be painted on the metal canal replica with a fine brush prior to pouring resin into the mould. The use of a vibrator is also helpful in removing air bubbles during the pouring process. Resin is then allowed to polymerise at room temperature.

\section{Finishing}

Once the resin has set, the canal template is removed from the resin blocks using a pair of pliers that grips the coronal portion of the template. The small cube of agar hydrocolloid, silicone or wax at the end of each canal is also removed and a K-file size 8 is passed through the canal to ensure patency. No polishing is required, as the four surfaces of the cuvettes are highly polished and clear (Figures $2 \mathrm{~d}$ and 4). The polymerised resin block that is constructed with a silicone mould has an opaque and slightly rough surface, which may be smoothened and rendered transparent by polishing with sandpaper, pumice and whitening. A funnel or saucer shaped well is formed with a bur at the top of the canal to simulate the canal orifice.

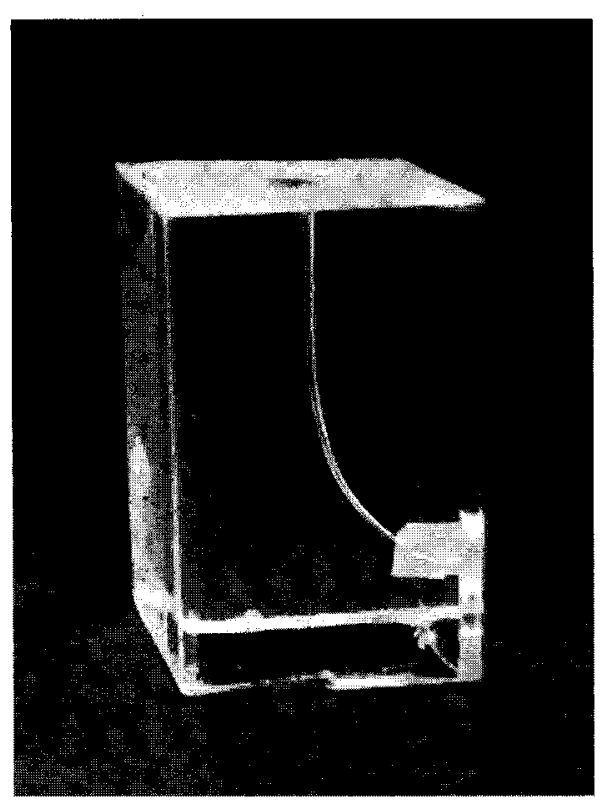

Figure 4: A resin block with a simulated curved canal produced in the Faculty. 
Although there are some limitations, RSC is still a valuable aid for teaching pre-clinical endodontics. The method described above is easy, inexpensive and can produce a large quantity of standardised RSC.

\section{ACKNOWLEDGEMENT}

We would like to acknowledge Encik Mohd. Abd. Rahim Bahari for his technical assistance in the development of the method of constructing RSC.

\section{REFERENCES}

1. Weine FS, Kelly RF, Lio PJ. The effect of preparation procedures on original canal shape and on apical foramen shape. J Endod 1975; 1:25562.

2. Spenst A, Kahn H. The use of a plastic block for teaching root canal instrumentation and obturation. J Endod 1979; 5:282-4.

3. LaTurno SA, Corcoran JF, Ellison RL. An evaluation of a teaching aid in endodontics. $J$ Endod 1984; 10:507-11.
4. Lim KC, Webber $\mathbf{J}$. The validity of simulated root canals for the investigation of the prepared root canal shape. Int Endod J 1985; 18:240-6.

5. Schulz-Bongert U, Weine FS. Method for constructing standardized simulated root canals. J Dent Educ 1990; 54:328-30.

6. Dummer PM, Alodeh MH, Al-Omari MA. A method for the construction of simulated root canals in clear resin blocks. Int Endod J 1991; 24:63-6.

7. Melton DC, Krell KV, Fuller MW. Anatomical and histological features of C-shaped canals in mandibular second molars. J Endod 1991; 17:3848.

8. Alodeh MH, Doller R, Dummer PM. Shaping of simulated root canals in resin blocks using the step-back technique with $\mathrm{K}$-files manipulated in a simple in out filing motion. Int Endod J 1989; 22:107-17.

9. Skidmore AE, Bjorndal AM. Root canal morphology of the human mandibular first molar. Oral Surg 1971; 32:119-241. 